\title{
STABILITY AND UNIQUENESS FOR A TURBULENCE MODEL OF BURGERS*
}

\author{
C. O. HORGAN ${ }^{1}$ AND W. E. OLMSTEAD ${ }^{2}$ (Northwestern University)
}

\begin{abstract}
In his early work on mathematical models of turbulence, J. M. Burgers proposed a nonlinear system, coupling an ordinary and a partial differential equation, to simulate flow in a channel. The now well-known Burgers equation arose in his work from a simplification of this system. The original system has some interesting features not shared by the Burgers equation. This investigation establishes results on the stability of the "laminar" stationary solution and uniqueness of the nonstationary solution of the system.
\end{abstract}

1. Introduction. The quasilinear parabolic equation

$$
\frac{\partial V}{\partial t}+V \frac{\partial V}{\partial y}=\frac{1}{R} \frac{\partial^{2} V}{\partial y^{2}}
$$

is known as the Burgers equation owing to its appearance in the work of J. M. Burgers [1, 2] on mathematical models of turbulence. This equation has also been associated with other physical phenomena of fluid dynamics, and numerous papers have been devoted to solving it. An extensive survey of the literature relating to (1) can be found in Benton and Platzman [3].

From Burgers' work, it is clear that (1) arises from a simplification of a system of two equations. This Burgers system for $U(t)$ and $v(y, t)$ is given in nondimensional form by

$$
\begin{gathered}
\frac{d U}{d t}=P-\frac{1}{R} U-\int_{0}^{1} v^{2} d y, \\
\frac{\partial v}{\partial t}+2 v \frac{\partial v}{\partial y}=\frac{1}{R} \frac{\partial^{2} v}{\partial y^{2}}+U v .
\end{gathered}
$$

This system was one of several mathematical models for turbulence proposed by Burgers [1]. It was intended to portray flow in a channel $(0 \leq y \leq 1)$. In the model, $U(t)$ denotes the principal velocity in the channel due to some applied force $P$, while $v(y, t)$ signifies the turbulent perturbation of the motion. In this nondimensional form, the spatial variable has been normalized by the channel width $b$, and both velocities by some characteristic speed $U_{0}$ of the principal flow. Then $R=b U_{0} / \nu$ takes the role of a Reynolds number for a given kinematic viscosity $\nu$ of the fluid.

A loose interpretation of this model suggests that $(2.1)$ is a balance between the acceleration of the principal flow and the applied force, viscous damping, and turbulent damping (integral term). In (2.2), the acceleration of the turbulent motion is balanced by viscous diffusion and convective coupling with the principal flow.

\footnotetext{
* Received November 18, 1977.

${ }^{1}$ On leave from University of Houston. This author's work was supported by the National Science Foundation under Grant ENG 75-13643.

${ }^{2}$ This author's work was supported by the National Science Foundation under Grant MCS 77-01327.
} 
The Burgers equation (1) follows from a simplification of (2.2) suggested in [1] with $U$ $\equiv 0, v \equiv \frac{1}{2} V$. Of course this reduction generally ignores the satisfaction of (2.1). Consequently, it is not surprising that the Burgers system exhibits some features which are entirely different from the Burgers equation. For example, the only stationary solution of (1) which vanishes at $y=0,1$ is $V \equiv 0$; whereas (2) has nontrivial solutions which arise as bifurcations at $P R^{2}=n^{2} \pi^{2}, n=1,2, \cdots$.

Our investigation here will focus on stability and uniqueness questions for the Burgers system. In our analysis we prefer to consider an equivalent form of (2) suggested by Eckhaus [4]. That form is achieved by taking

$$
u(t)=U(t)-\frac{\lambda}{R}, \quad \lambda=P R^{2}, \quad P \geq 0,
$$

so that the Burgers system becomes

$$
\begin{gathered}
\frac{d u}{d t}+\frac{1}{R} u=-\int_{0}^{1} v^{2} d y, \\
\frac{\partial v}{\partial t}=\frac{1}{R} \frac{\partial^{2} v}{\partial y^{2}}+\frac{\lambda}{R} v-2 v \frac{\partial v}{\partial y}+u v, \quad 0<y<1, \quad t>0,
\end{gathered}
$$

with boundary and initial conditions assigned as

$$
v(0, t)=v(1, t)=0, \quad v(y, 0)=v_{0}(y), \quad u(0)=u_{0} .
$$

2. Stationary solutions. To study the time-independent solutions of (4), we set

$$
v(y, t)=\frac{1}{R} \varphi(y), \quad u(t)=-\frac{1}{R} \int_{0}^{1} \varphi^{2} d y,
$$

so that (4.1) is satisfied, while (4.2) leads to the nonlinear eigenvalue problem

$$
\begin{gathered}
\frac{d^{2} \varphi}{d y^{2}}-2 \varphi \frac{d \varphi}{d y}+\left[\lambda-\int_{0}^{1} \varphi^{2}(\eta) d \eta\right] \varphi=0, \quad 0<y<1, \\
\varphi(0)=\varphi(1)=0 .
\end{gathered}
$$

The trivial solution $\varphi \equiv 0$, corresponding to "laminar flow" $(v \equiv 0, U \equiv \lambda / R)$, satisfies (7) for all $\lambda$. We can easily show that this is the only solution of (7) for $\lambda<\pi^{2}$. Upon multiplication of (7.1) by $\varphi$, integration over the interval of interest, and utilization of (7.2), we find

$$
\lambda \int_{0}^{1} \varphi^{2} d y-\left(\int_{0}^{1} \varphi^{2} d y\right)^{2}=\int_{0}^{1}\left(\frac{d \varphi}{d y}\right)^{2} d y \geq \pi^{2} \int_{0}^{1} \varphi^{2} d y,
$$

where we have used a standard inequality of Wirtinger type in the final step. We next express (8) as

$$
\left(\int_{0}^{1} \varphi^{2} d y\right)\left[\int_{0}^{1} \varphi^{2} d y+\left(\pi^{2}-\lambda\right)\right] \leq 0,
$$

which must hold for all solutions of (7). If $\lambda<\pi^{2}$, it is readily seen that only $\varphi \equiv 0$ can satisfy (9).

There are nontrivial solutions of (7) which bifurcate from $\varphi \equiv 0$ at each of the eigenvalues $\lambda_{n}=n^{2} \pi^{2}, n=1,2, \cdots$ of the linearized version of (7). We can deduce the 
nature of these solutions near the eigenvalues by the following formal procedure. For a given eigenvalue $\lambda_{n}$, rewrite (7.1) as

$$
\frac{d^{2} \varphi}{d y^{2}}+\lambda_{n} \varphi=2 \varphi \frac{d \varphi}{d y}+\left[\left(\lambda_{n}-\lambda\right)+\int_{0}^{1} \varphi^{2}(\eta) d \eta\right] \varphi, \quad 0<y<1,
$$

and seek a solution which has the asymptotic form

$$
\varphi=A \sin \sqrt{ } \lambda_{n} y+o(A)
$$

where $A \rightarrow 0$ as $\lambda \rightarrow \lambda_{n}$, and which also satisfies the boundary conditions (7.2). Then, the alternative theorem for boundary value problems requires that the right side of (10) be orthogonal to the eigenfunction $\sin \sqrt{ } \lambda_{n} y$. Thus, inserting (11) into the right side of (10), multiplying the $\sin \sqrt{ } \lambda_{n} y$, and integrating over the indicated interval yields

$$
A= \pm\left[2\left(\lambda-\lambda_{n}\right)\right]^{1 / 2}, \quad \lambda>\lambda_{n} .
$$

Similar calculations to demonstrate these nontrivial solutions were carried out by Burgers [1, 2] and Eckhaus [4]. The results suggest the existence of some nontrivial stationary solutions for all $\lambda>\lambda_{1}=\pi^{2}$. Such solutions would correspond to turbulent states of the flow, and suggest that the laminar state $\varphi \equiv 0$ would never be stable for $\lambda>$ $\pi^{2}$.

3. Stability. Here we establish the asymptotic stability of the "laminar" stationary solution $(u \equiv 0, v \equiv 0)$ of (4.1) and (4.2). Our arguments involve the energy equation associated with the Burgers system (4). From (4), it is straightforward to obtain

$$
\begin{gathered}
\frac{1}{2} \frac{d u^{2}}{d t}+\frac{1}{R} u^{2}=-u E, \\
\frac{1}{2} \frac{d E}{d t}=-\frac{1}{R} \int_{0}^{1}\left(\frac{\partial v}{\partial y}\right)^{2} d y+\frac{\lambda}{R} E+u E, \quad t>0,
\end{gathered}
$$

where

$$
E(t) \equiv \int_{0}^{1} v^{2}(y, t) d y \geq 0 ; E_{0} \equiv E(0)=\int_{0}^{1} v_{0}^{2} d y
$$

The desired energy equation then follows from (13) as

$$
\frac{1}{2} \frac{d}{d t}\left(E+u^{2}\right)=-\frac{1}{R} u^{2}+\frac{\lambda}{R} E-\frac{1}{R} \int_{0}^{1}\left(\frac{\partial v}{\partial y}\right)^{2} d y, \quad t>0 .
$$

An equivalent form of (15) was considered by Burgers [1], and indeed its character seems to have provided some of his motivation for the system (2) as a model of turbulence.

By again using the Wirtinger inequality employed in (8) we deduce from (15) the differential inequality

$$
\frac{1}{2} \frac{d}{d t}\left(E+u^{2}\right) \leq-\frac{1}{R} u^{2}-\left(\frac{\pi^{2}-\lambda}{R}\right) E, \quad t>0 .
$$

Golia and Abel [5] have considered the system (4) as an example illustrating their method for constructing Lyapunov functionals. They obtained (16) and noted that if $\lambda<\pi^{2}$, then the Lyapunov functional $\left(E+u^{2}\right)$ is nonincreasing, hence implying stability for the "laminar" or trivial stationary solution. 
This result can be improved by observing that (16) may be expressed as

$$
\left(\frac{d}{d t}+\frac{2 k}{R}\right)\left(E+u^{2}\right) \leq-2\left[\left(\frac{1-k}{R}\right) u^{2}+\left(\frac{\pi^{2}-\lambda-k}{R}\right) E\right]
$$

where

$$
k=\min \left\{1, \pi^{2}-\lambda\right\}, \quad \lambda<\pi^{2} .
$$

Clearly $k>0$ and

$$
\left(\frac{d}{d t}+\frac{2 k}{R}\right)\left(E+u^{2}\right) \leq 0
$$

which can be integrated to yield

$$
E(t)+u^{2}(t) \leq\left(E_{0}+u_{0}^{2}\right) \exp \left(-\frac{2 k t}{R}\right), \quad t \geq 0 .
$$

Thus for $\lambda<\pi^{2}$, (20) establishes that the Burgers system is exponentially asymptotically stable with respect to the "laminar" stationary solution $(u \equiv 0, v \equiv 0)$.

Since the bound (20) applies to the sum $E+u^{2}$ with a decay constant which changes form at $\lambda=\pi^{2}-1$, it is natural to inquire about the individual behavior of $E$ and $u^{2}$ in the stability question. To study $E$, we obtain from (13.2) that

$$
\frac{1}{2} \frac{d E}{d t} \leq\left(\frac{\lambda-\pi^{2}}{R}\right) E+u E
$$

which can be integrated to yield

$$
E(t) \leq E_{0} \exp \left(-\frac{2\left(\pi^{2}-\lambda\right) t}{R}\right) \exp \left(2 \int_{0}^{t} u(\tau) d \tau\right) .
$$

We can also solve (4.1) for $u$ in terms of $E$ to obtain

$$
u(t)=u_{0} \exp (-t / R)-\exp (-t / R) \int_{0}^{t} \exp (\tau / R) E(\tau) d \tau \leq u_{0} \exp (-t / R), \quad t \geq 0 .
$$

Utilizing this upper bound in (22) provides the estimate

$$
E(t) \leq \gamma E_{0} \exp \left(-\frac{2\left(\pi^{2}-\lambda\right) t}{R}\right), \quad \gamma=\min \left\{1, \exp \left(2 R u_{0}\right)\right\} .
$$

We can then employ this result to obtain a lower bound on $u$ from (23) as

$$
u(t) \geq \exp (-t / R)\left[u_{0}-\gamma E_{0} Q(t)\right]
$$

where

$$
Q(t)=\frac{R}{2\left(\pi^{2}-\lambda\right)-1}\left[1-\exp \left(\frac{1-2\left(\pi^{2}-\lambda\right) t}{R}\right)\right]
$$

Here it is understood that the limiting form $Q=t$ is taken if $\lambda=\pi^{2}-\frac{1}{2}$.

It is worthwhile to develop two separate cases in (25). 
We find that

$$
\begin{aligned}
& {\left[u_{0}-\frac{\gamma E_{0} R}{2\left(\pi^{2}-\lambda\right)-1}\right] \exp (-t / R) \leq u(t) \leq u_{0} \exp (-t / R), \quad \lambda<\pi^{2}-\frac{1}{2},} \\
& u_{0} \exp (-t / R)-\frac{\gamma E_{0} R}{1-2\left(\pi^{2}-\lambda\right)} \exp \left(-\frac{2\left(\pi^{2}-\lambda\right) t}{R}\right) \\
& \leq u(t) \leq u_{0} \exp (-t / R), \quad \lambda>\pi^{2}-\frac{1}{2}
\end{aligned}
$$

In (26.1) the upper and lower bounds have the same exponentially decaying factor, although the multiplicative constant in the lower bound is of undetermined sign when $u_{0}>$ 0 . However if $0 \leq \gamma E_{0} R / 2\left(\pi^{2}-\lambda\right)-1<u_{0}$, then (26.1) implies that $u \geq 0$ and furnishes meaningful bounds. In (26.2) the lower bound is eventually dominated by the second term, and hence must ultimately be negative even if $u_{0}>0$. Moreover, it seems likely that this behavior can actually be realized. Indeed, we can show that if $u$ is ever zero, then it can never be positive at any later time. This is easily seen by integrating (4.1) to obtain

$$
u(t)=\exp \left(\underline{t_{0}} \frac{-t}{R}\right) u\left(t_{0}\right)-\exp (-t / R) \int_{t_{0}}^{t} \exp (\tau / R) E(\tau) d \tau, \quad t \geq t_{0}
$$

from which follows

$$
u(t) \leq 0 \text { for } t>t_{0} \text { if } u\left(t_{0}\right)=0 \text {. }
$$

These results on stability do suggest some interpretation of the Burgers system. According to (24) the decay of the turbulent energy $E$ is governed by how large $\lambda=P R^{2}$ is compared to the critical value $\pi^{2}$. For $\lambda$ sufficiently small, the turbulent energy will decay faster than the energy $u^{2}$ associated with variations in the principal flow, which is governed by viscous damping as seen in (26.1). However, for larger $\lambda$ near the critical value, the turbulent energy decays much more slowly, and through convective interaction it can inhibit the decay of any variations in the principal flow, as suggested by the lower bound in (26.2). These observations are compatible with the bound (20) on the total energy.

4. Uniqueness. Here we establish that there is at most one classical solution of the nonstationary Burgers system (4) subject to the boundary and initial conditions (5). Moreover, this uniqueness holds for all value of $\lambda$.

Suppose there are two solutions $\left(u_{1}, v_{1}\right)$ and $\left(u_{2}, v_{2}\right)$ satisfying $(4)$ and (5). We set

$$
z(t) \equiv u_{1}(t)-u_{2}(t), \quad w(y, t) \equiv v_{1}(y, t)-v_{2}(y, t) .
$$

It then follows from (4) and (5) that $z(t)$ and $w(y, t)$ must satisfy

$$
\begin{gathered}
\frac{d z}{d t}+\frac{1}{R} z=-\int_{0}^{1}\left(v_{1}+v_{2}\right) w d y \\
\frac{\partial w}{\partial t}=\frac{1}{R} \frac{\partial^{2} w}{\partial y^{2}}+\frac{\lambda}{R} w-\frac{\partial}{\partial y}\left[\left(v_{1}+v_{2}\right) w\right]+\frac{1}{2}\left(u_{1}+u_{2}\right) w+\frac{1}{2}\left(v_{1}+v_{2}\right) z
\end{gathered}
$$

with homogeneous boundary and initial conditions

$$
w(0, t)=w(1, t)=0, \quad w(y, 0)=0, \quad z(0)=0 .
$$


From (30) we deduce that

$$
\begin{aligned}
& \frac{1}{2} \frac{d z^{2}}{d t}+\frac{1}{R} z^{2}=-z \int_{0}^{1}\left(v_{1}+v_{2}\right) w d y \\
& \frac{1}{2} \frac{d}{d t} \int_{0}^{1} w^{2} d y=- \frac{1}{R} \int_{0}^{1}\left(\frac{\partial w}{\partial y}\right)^{2} d y+\frac{\lambda}{R} \int_{0}^{1} w^{2} d y-\frac{1}{2} \int_{0}^{1} w^{2} \frac{\partial}{\partial y}\left(v_{1}+v_{2}\right) d y \\
&+\frac{1}{2}\left(u_{1}+u_{2}\right) \int_{0}^{1} w^{2} d y+\frac{z}{2} \int_{0}^{1}\left(v_{1}+v_{2}\right) w d y
\end{aligned}
$$

Then we introduce

$$
I(t) \equiv \int_{0}^{1} w^{2}(y, t) d y+\frac{1}{2} z^{2}(t) \geq 0, \quad I_{0} \equiv I(0)=0
$$

and combine $(32)$ to find

$$
\begin{aligned}
\frac{d I(t)}{d t}= & -\frac{1}{R} z^{2}+\left(\frac{2 \lambda}{R}+u_{1}+u_{2}\right) \int_{0}^{1} w^{2} d y \\
& -\frac{2}{R} \int_{0}^{1}\left(\frac{\partial w}{\partial y}\right)^{2} d y-\int_{0}^{1} w^{2} \frac{\partial}{\partial y}\left(v_{1}+v_{2}\right) d y .
\end{aligned}
$$

Before we can proceed, we must obtain estimates for some quantities appearing in (34).

Since $u_{1}$ and $u_{2}$ both satisfy (4.1), we find that

$$
\begin{aligned}
\frac{d}{d t}\left(u_{1}+u_{2}\right)+ & \frac{1}{R}\left(u_{1}+u_{2}\right)=-\int_{0}^{1}\left(v_{1}^{2}+v_{2}^{2}\right) d y \leq 0, \\
u_{1}(0)+u_{2}(0) & =2 u(0),
\end{aligned}
$$

from which we obtain

$$
u_{1}(t)+u_{2}(t) \leq 2 u(0) \exp (-t / R), \quad t \geq 0 .
$$

Since $v_{1}$ and $v_{2}$ are assumed to be classical solutions, then $\partial\left(v_{1}+v_{2}\right) / \partial y$ is smooth enough to assert that there exists some $\theta(t)$ such that

$$
\int_{0}^{1} w^{2} \frac{\partial}{\partial y}\left(v_{1}+v_{2}\right) d y=\theta(t) \int_{0}^{1} w^{2} d y .
$$

Incorporating (36) and (37) into (34) we obtain the differential inequality

$$
\frac{d I}{d t} \leq\left[2\left(\frac{\lambda}{R}+u_{0} \exp (-t / R)\right)+|\theta|\right] I, \quad t>0 .
$$

Integration of this and utilizing $I_{0}=0$ yields

$$
I(t) \leq 0, \quad t \geq 0,
$$

which can only be satisfied if

$$
z(t) \equiv 0, \quad w(y, t) \equiv 0, \quad 0 \leq y \leq 1, \quad t \geq 0,
$$

thereby establishing uniqueness. 


\section{REFERENCES}

[1] J. M. Burgers, Mathematical examples illustrating relations occurring in the theory of turbulent fluid motion, Trans. Roy. Neth. Acad. Sci. Amsterdam 17, 1-53 (1939)

[2] J. M. Burgers, A mathematical model illustrating the theory of turbulence, in Advances in Applied Mechanics (R. von Mises and T. von Kármán, editors) 1, Academic Press, New York, 1948, pp. 171-199

[3] E. R. Benton and G. W. Platzman, A table of solutions of the one-dimensional Burgers equation, Quart. Appl. Math. 30, 195-212 (1972)

[4] W. Eckhaus, Studies in non-linear stability theory, Springer Tracts in Natural Philosophy, Vol. 6, SpringerVerlag, Berlin, 1965

[5] C. Golia and J. M. Abel, Path integral synthesis of Lyapunov functionals for partial differential equations, Int. J. Non-Linear Mech. 10 333-344 (1975) 